\title{
Arbeitsmedizinische Aspekte in der Allgemein-(Viszeral-)Chirurgie - Infektionsgefährdung durch Nadelstichverletzungen (was der Chirurg wissen sollte)
}

\author{
Occupational Medicine Aspects in General and Abdominal Surgery - \\ Risk of Infection Attributable to Needlestick Injuries (What the Surgeon should Know)
}

Autoren

Institute
S. Darius ${ }^{1}$, F. Meyer ${ }^{2}$, I. Boeckelmann ${ }^{1}$

${ }^{1}$ Bereich Arbeitsmedizin, Otto-von-Guericke-Universitätsklinikum Magdeburg A. ö.R, Deutschland

${ }^{2}$ Klinik für Allgemein-, Viszeral- und Gefäßchirurgie, Otto-von-Guericke-Universitätsklinikum Magdeburg A. ö. R, Deutschland

\author{
Schlüsselwörter \\ - Nadelstichverletzung \\ - Infektionsrisiko \\ - Präventionsmaßnahmen \\ Key words \\ - needle stick injury \\ - infection risk \\ - preventative measures
}

\section{Bibliografie}

DOI http://dx.doi.org/

10.1055/s-0032-1315202

Zentralbl Chir 2013; 138:

88-93 @ Georg Thieme

Verlag KG Stuttgart · New York .

ISSN 0044-409X

Korrespondenzadresse Dr. med. Sabine Darius

Bereich Arbeitsmedizin

Otto-von-Guericke-Universitäts-

klinikum A. ö. R

Leipziger Straße 44

39120 Magdeburg

Tel.: 0391/67-15057

Fax: 0391/67-15083

sabine.darius@med.ovgu.de

\section{Zusammenfassung \\ $\nabla$}

Zu den häufigsten Arbeitsunfällen bei Mitarbeitern im Gesundheitswesen zählen Stich- und Schnittverletzungen, was damit eine immense arbeitsmedizinische Dimension aufweist. Das Ziel dieser Arbeit ist es, eine aktuelle Übersicht über das Infektionsrisiko im OP-Saal bei den wichtigsten Erregern zu erstellen und arbeitsmedizinische Empfehlungen für technische, organisatorische und persönliche Arbeitsschutzmaßnahmen für das OP-Personal sowie zur Postexpositionsprophylaxe nach Verletzungen zu geben. Des Weiteren werden einige versicherungsrechtliche Aspekte zu Berufskrankheiten dargestellt. Sind die Instrumente, mit denen sich die Beschäftigten verletzen, mit Blut oder anderen Körperflüssigkeiten kontaminiert, besteht das Risiko einer Infektion. Die wichtigsten Erreger, die durch Blutkontakt übertragen werden, sind das HepatitisB-Virus (HBV), das Hepatitis-C-Virus (HCV) und das Human Immunodeficiency Virus (HIV). Das Risiko der Ansteckung hängt sowohl vom Infektionsstatus des Indexpatienten ab als auch vom Immunstatus des exponierten Mitarbeiters. Weiterhin spielen Art und Schwere der Verletzung, die kontaminierende Menge Blut, die Dauer des Blutkontakts sowie das Zeitintervall zwischen Verletzung und Reinigung und der Anwendung von Postexpositionsmaßnahmen eine Rolle. Arbeitsmedizinisch ist die Prävention vordringlich, wobei stets Ziel sein sollte, sich keine Nadelstichverletzung (NSV) zuzuziehen und sich so vor Infektionen zu schützen. Die Verwendung von sicheren Instrumenten bei invasiven Eingriffen sowie das Tragen von doppelten Handschuhen für eine verstärkte Sicherheit beim Umgang mit spitzen und scharfen Gegenständen sollten zur Selbstverständlichkeit beim medizinischen Personal nicht nur im operativen Bereich werden.

\section{Abstract \\ $\nabla$}

Needlestick, stab, scratch, and cut injuries are a common problem and a significant health hazard among healthcare workers. The aim of this review is to give an overview on the risk of infection in general, abdominal and vascular surgery and to suggest occupational safety measures. Furthermore, we want to discuss insurance-related aspects. If medical devices are contaminated with blood or other body fluids, there is a relevant risk of infection with hepatitis B virus (HBV), hepatitis $\mathrm{C}$ virus (HCV), and human immunodeficiency virus (HIV). The risk of transmission depends on the infection status of the patient, and on the immune status of the healthcare worker. In addition, the risk of infection is affected by the type and severity of injuries, by the quantity (volume) of blood, the time between injury and cleaning, and the administration of post-exposure prophylaxis. Prevention measures are an important focus in occupational medicine. Comprehensive programmes to prevent injuries (usage of safety devices, surgical gloves, and of disposal containers) have to be continuously considered to minimize risk of infection of healthcare workers. 


\section{Einleitung}

$\nabla$

Zu den häufigsten Arbeitsunfällen bei Mitarbeitern im Gesundheitswesen zählen Verletzungen an spitzen und scharfen Gegenständen, die auch gleichzeitig eine der größten Gefahren darstellen. Dabei entstehen gesamtwirtschaftliche Kosten in Höhe von rund 47 Mio. EUR [1]. Unter dem Begriff Nadelstichverletzung (NSV) werden im Besonderen jegliche Stich-, Schnitt- und Kratzverletzungen der Haut durch Nadeln, Skalpelle, spitze und scharfe Gegenstände zusammengefasst, die mit Patientenblut oder anderem potenziell infektiösen Material verunreinigt waren oder sein können - unabhängig davon, ob die Wunde geblutet hat oder nicht. Schätzungen zufolge kommt es in Deutschland zu etwa 500000 NSV pro Jahr, wobei Expertenmeinungen zufolge nur etwa 10-20\% aller Verletzungen angezeigt werden [2]. Chirurgen sind während der Ausübung ihres Berufs dabei einem besonderen Risiko ausgesetzt, sich an kontaminierten Instrumenten mit Krankheitserregern zu infizieren, die durch Kontakt mit infektiösem Blut oder anderen Flüssigkeiten übertragen werden [3]. Durch Unachtsamkeit kann es beim Weiterreichen von Instrumenten von Hand zu Hand während der Operation bzw. beim Entsorgen der Instrumente nach der OP zu einer Verletzung des Personals kommen. Laut einer Umfrage werden diese Unfälle hauptsächlich durch Unaufmerksamkeit infolge von (zu) hoher Stressbelastung sowie durch Nichteinhalten von Vorschriften (z.B. „Recapping“) verursacht. Teilweise spielte auch eine Rolle, dass die Vorschriften selbst unzureichend waren oder auch der Arbeitsablauf an sich ein sicheres Arbeiten nicht zuließ [2]. In der Chirurgie war entsprechend der Frankfurter Nadelstichstudie $[3,4]$ die Rate der NSV besonders hoch. Als ein Risikofaktor wurde mit 28,6\% das Nähen ermittelt, gefolgt von Schnittverletzungen während der Operation (12,5\%) und Stichverletzungen bei Blutentnahmen. Aber auch intraoperative Verletzungen durch Knochensplitter spielten als „Sonstige Verletzung“ eine nicht unerhebliche Rolle.

Das Ziel dieser Arbeit ist es, basierend auf einer repräsentativen Literaturrecherche und eigener arbeitsmedizinisch-klinischer Erfahrungen eine aktuelle Übersicht über das Infektionsrisiko im OP-Saal bei den wichtigsten Erregern zu erstellen und arbeitsmedizinische Empfehlungen für technische, organisatorische und persönliche Arbeitsschutzmaßnahmen für das OP-Personal sowie zur Postexpositionsprophylaxe nach Verletzungen zu ge- ben. Des Weiteren werden einige versicherungsrechtliche Aspekte zu Berufskrankheiten dargestellt.

\section{Grundlagen \\ $\nabla$}

Sind die Instrumente, mit denen sich die operativ tätigen Mitarbeiter verletzen, mit Blut oder anderen Körperflüssigkeiten kontaminiert, besteht das Risiko einer Infektion. Die wichtigsten Erreger, die durch Blutkontakt übertragen werden, sind das Hepatitis-B-Virus (HBV), das Hepatitis-C-Virus (HCV) und das Human Immunodeficiency Virus (HIV). Aber auch die Übertragung anderer Mikroorganismen durch NSV ist möglich. So sind in der Literatur Fälle beschrieben worden, bei denen Viren (z. B. Ebola- oder Dengue-Viren, Herpes-simplex-Virus), Bakterien (z.B. Mycobacterium tuberculosis, Staphylococcus aureus), Pilze, Protozoen (Plasmodium falciparum, Toxoplasma gondii) und auch Prionen durch Blut von Patienten auf Mitarbeiter infolge NSV übertragen wurden [5].

Diese Infektionserkrankungen können als Berufskrankheit (BK 3101) anerkannt werden, wenn der Mitarbeiter im Gesundheitsdienst, in der Wohlfahrtspflege oder in einem Laboratorium tätig oder durch eine andere Tätigkeit der Infektionsgefahr in ähnlichem Maße besonders ausgesetzt war. Im Jahr 2006 wurden laut Statistik der Deutschen Gesetzlichen Unfallversicherung (DGUV) noch über 4700 Verdachtsanzeigen auf Anerkennung der BK 3101 gestellt, von denen 537 Fälle tatsächlich als Berufskrankheit BK 3101 anerkannt worden sind. Im Jahr 2010 wurden nur noch knapp 1500 Verdachtsanzeigen gestellt, von denen 579 Fälle als BK anerkannt wurden [6,7]. Die Zahl der Fälle, bei denen neue Renten gezahlt wurden, ist seit 2006 rückläufig ( $\bullet$ Abb. 1).

Es ist jedoch schwierig, diese Zahlen nochmals in die verschiedenen Tätigkeitsfelder im Gesundheitsdienst zu unterteilen. So ist z.B. die Zuordnung der BK 3101 zum Tätigkeitsfeld im OP-Saal kaum möglich, da Mitarbeiter nicht nur dort tätig sind und die Ansteckungsgefahr auch auf den Stationen (z.B. bei Blutentnahmen) hoch ist.

Da die Infektionskrankheiten auch außerberuflich übertragen werden können, ist sowohl aus arbeitsmedizinischer als auch aus versicherungsrechtlicher Sicht die Meldung jeder NSV beim Betriebsarzt oder dem zuständigen D-Arzt notwendig. Nur wenn die NSV sorgfältig dokumentiert und somit der Nachweis einer

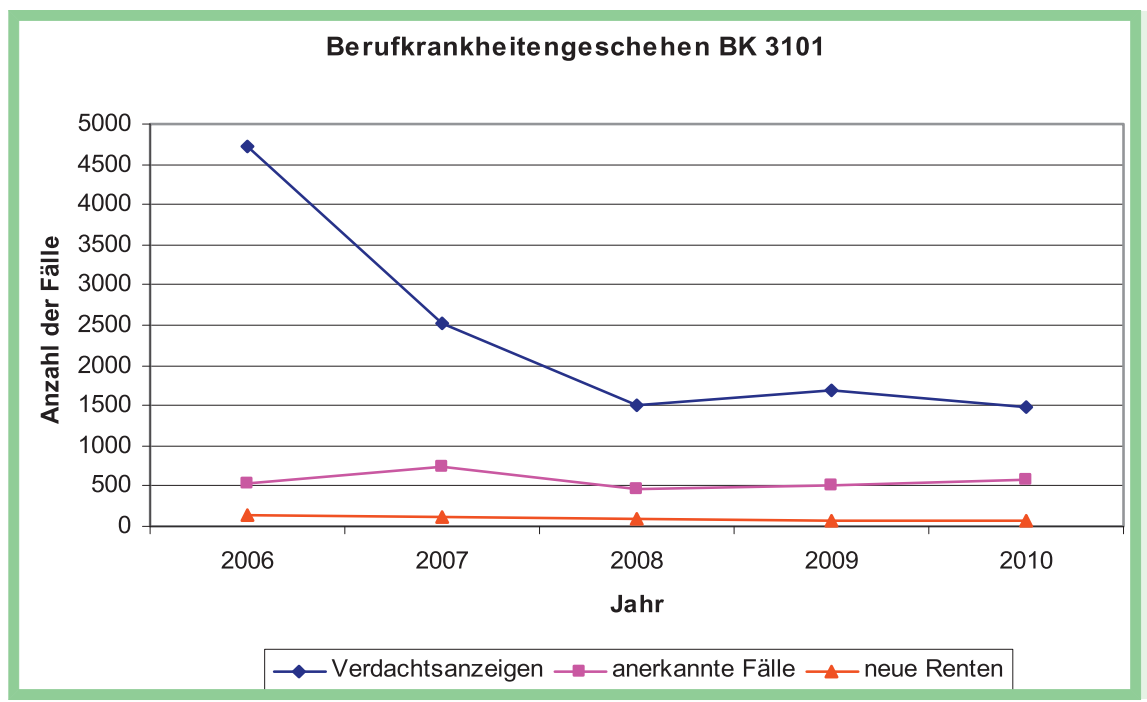

Abb. 1 DGUV-Statistik zum Berufskrankheitengeschehen der BK 3101 - Infektionskrankheiten, wenn der Mitarbeiter im Gesundheitsdienst, in der Wohlfahrtspflege oder in einem Laboratorium tätig oder durch eine andere Tätigkeit der Infektionsgefahr in ähnlichem Maße besonders ausgesetzt war (Quelle: DGUV-Statistiken für die Praxis 2010; http://www.dguv.de/inhalt/zahlen/documents/ dguvstatistiken2010d.pdf, [7]). 
haftungsausfüllenden und -begründenden Kausalität erbracht wurde, besteht Aussicht auf Anerkennung der Infektionserkrankung als Berufserkrankung. Eine haftungsausfüllende Kausalität liegt vor, wenn

- nach den aktuellen Erkenntnissen die Art und Intensität des einwirkenden Faktors dieser als „wesentliche Bedingung“ für das Zustandekommen der Erkrankung angesehen werden kann,

- der Erkrankte in einer versicherten Tätigkeit beschäftigt war und

- der als ursächlich angesehene Faktor bei dieser Tätigkeit auch nachweislich eingewirkt hat.

Kann dieser Nachweis nicht erbracht werden (z.B. wegen fehlender Dokumentation), ist die Anerkennung der Infektionserkrankung als BK schwierig durchzusetzen, was auch die Differenz zwischen den Verdachtsanzeigen und den tatsächlich anerkannten Fällen erklärt.

\section{Infektionsrisiko}

$\nabla$

Das Risiko der Ansteckung hängt sowohl vom Infektionsstatus des Indexpatienten als auch vom Immunstatus des exponierten Mitarbeiters ab. Weiterhin spielen Art und Schwere der Verletzung, die kontaminierende Menge Blut, die Dauer des Blutkontakts sowie das Zeitintervall zwischen Verletzung und Reinigung und der Anwendung von Postexpositionsmaßnahmen eine Rolle.

\section{Hepatitis B}

Das Hepatitis-B-Virus ist weltweit verbreitet. Schätzungen zufolge haben in Deutschland etwa 5-8\% der Bevölkerung eine Hepatitis-B-Infektion durchgemacht. Die Zahl chronischer HBs-Antigen-Träger wird auf $0,4-0,8 \%$ geschätzt $[8,9]$. Das Übertragungsrisiko liegt je nach Infektionsstatus des Indexpatienten und Konzentration des Erregers zwischen 30 und 100\% [4,10-12].

Nach Angaben des Robert Koch-Instituts erreichen Hepatitis-BViren insbesondere im Blut sehr hohe Konzentrationen von bis zu $10^{11}$ Viruspartikel/ml Serum und über $10^{8}$ Viruspartikel $/ \mathrm{ml}$ infektiöse Einheiten. In der Frühphase der Infektion und bei HBeAg-positiven HBV-Trägern ist fast jedes Viruspartikel infektiös, in der Spätphase und bei HBeAg-negativen Trägern dagegen meist nur jedes 100ste bis 1000ste Viruspartikel. Demzufolge können in der Frühphase und bei HBeAg-positiven Trägern auch kleinste Mengen Blut eine Infektion hervorrufen, selbst wenn die Verletzung geringfügig scheint [13]. In Versuchen konnte nachgewiesen werden, dass selbst durch kleinste NSV große Blutmengen übertragen werden können [14].

Bei der Berufsgenossenschaft für Gesundheitsdienst und Wohlfahrtspflege (BGW) gingen im Jahr 2010 mit 71 Anzeigen einer vermutlich berufsbedingt erworbenen Hepatitis B in Deutschland weniger Anzeigen als im Jahr zuvor (75 Anzeigen) ein. In 31 Fällen wurde die Hepatitis B als Berufserkrankung anerkannt, wobei sich diese Fälle auf Erkrankungen aus verschiedenen Jahren beziehen [15]. Diese Statistiken beziehen sich jedoch nur auf die dort versicherten Personen und nicht auf Einrichtungen in kommunaler Trägerschaft und sind somit nicht vollständig. Die tatsächliche Zahl der als BK anerkannten Hepatitis-B-Fälle dürfte wesentlich höher liegen. In den letzten Jahren ist ein stetiger Rückgang der Verdachtsmeldungen von Hepatitis-B-Infektionen zu verzeichnen, was sehr wahrscheinlich auf die steigenden Impfraten und die verbesserte Sicherheit bei den Instrumenten zurückzuführen ist.

\section{Hepatitis C}

Ebenso wie der Erreger der Hepatitis B ist auch das Hepatitis-CVirus (HCV) weltweit verbreitet. Nach Schätzungen der WHO sind ca. 2-3\% der Weltbevölkerung mit HCV chronisch infiziert. In Deutschland sind ca. 0,2-0,5\% der Bevölkerung HCV-positiv erkrankt [15]. Die Infektion beginnt schleichend und unbemerkt. Etwa 20\% entwickeln eine Leberzirrhose [16], die als eine der häufigsten Indikationen für eine Lebertransplantation gilt. Bei einem Teil der Patienten kommt es zu einer karzinomatösen Entartung der Leberzirrhose. Lebensbedrohend sind auch Komplikationen wie die Dekompensation der Zirrhose mit Leberfunktionsstörung und Aszitesbildung, die hepatische Enzephalopathie und Ösophagus- oder Fundusvarizenblutungen [17].

Das Übertragungsrisiko im Gesundheitsdienst wird durchschnittlich mit ca. 2-3\% angegeben, einigen Autoren zufolge liegt das Risiko auch deutlich darunter [18-21]. Das Gefährdungspotenzial ist demnach deutlich niedriger als das von HepatitisB-Viren. Dennoch gingen bei der BGW in Hamburg im Jahr 2010 mit 100 Anzeigen einer vermutlich berufsbedingt erworbenen Hepatitis $C$ in Deutschland nahezu gleich viele Anträge wie im Jahr zuvor ein. In 45 Fällen wurde eine Hepatitis $C$ auch als BK anerkannt (diese Fälle beziehen sich wieder laut Angaben des Robert Koch-Instituts auf Erkrankungen aus verschiedenen Jahren [16]). Die Anzahl der tatsächlich als BK anerkannten HepatitisC-Fälle dürfte aber aufgrund der ähnlichen Problematik wie bei der Hepatitis B auch hier deutlich höher liegen.

\section{HIV}

Eine direkte Messung der HIV-Inzidenz bzw. der HIV-Prävalenz ist nicht möglich, sie kann nur mithilfe von Modellrechnungen abgeschätzt werden [22]. Die geschätzte Zahl der Menschen, die Ende 2011 in Deutschland mit HIV/AIDS leben, beträgt nach Angaben des Robert Koch-Instituts in etwa 73000, darunter 59000 Männer und 14000 Frauen.

Das durchschnittliche Risiko einer HIV-Serokonversion wird mit 0,3\% angegeben [10,12,23,24]. Das Risiko, sich bei HIV-positiven Patienten anzustecken, steigt jedoch um den Faktor 16 bei sehr tiefen Stich- oder Schnittverletzungen und noch um den Faktor 5, wenn sichtbare Blutspuren auf dem verletzenden Instrument vorhanden sind bzw. auch nach perkutaner Verletzung mit einer Injektionsnadel oder anderer Hohlraumnadel, die zuvor in Venen oder Arterien platziert waren. Bei hoher Viruslast im Blut des Indexpatienten (akute HIV-Infektion, AIDS im Endstadium) ist das Ansteckungsrisiko um das fünf- bis sechs-Fache erhöht [12,23, 24]. Insgesamt gesehen, ist das Risiko für das medizinische Personal, sich auf beruflichem Weg eine HIV-Infektion zuzuziehen, sehr gering. Bislang sind in Deutschland insgesamt nur 75 HIVInfektionen als Berufskrankheit anerkannt worden [25]. Wird eine medikamentöse Prophylaxe nach NSV mit kontaminierten Instrumenten durchgeführt, kann das Infektionsrisiko der exponierten Person deutlich gesenkt werden [12,24].

\section{Prävention}

$\nabla$

Durch die Vorschriften der Unfallversicherungen (früher berufsgenossenschaftliche Grundsätze) sind die Untersuchungen von Beschäftigten, die zeitlichen Untersuchungsabstände und das Methodeninventar für arbeitsmedizinische Vorsorgeuntersuchungen (z. B. G 42 - Tätigkeiten mit Infektionsgefährdung) geregelt. Arbeitsmedizinische Vorsorgeuntersuchungen dienen 
grundsätzlich der Früherkennung arbeitsbedingter Gesundheitsgefährdungen.

Der Schwerpunkt des Faches Arbeitsmedizin liegt in der Prävention von Arbeitsunfällen und Berufskrankheiten. Besonderes Augenmerk liegt dabei in der Primärprävention; es sollte nach Möglichkeit bereits vor einer Infektion, d.h. präexpositionell, alles für den Schutz und die Gesunderhaltung des Mitarbeiters getan werden. Fand trotz Vorsichtsmaßnahmen eine NSV mit Infektionsrisiko statt, so stehen im Fall von HBV und HIV Möglichkeiten der Postexpositionsprophylaxe bzw. der Sekundärprävention zur Verfügung.

\section{Primärprävention}

Durch technische, organisatorische und persönliche Maßnahmen muss die Übertragung von Krankheitserregern so weit wie möglich verhindert werden. Dabei haben technische Arbeitsschutzmaßnahmen stets Vorrang vor organisatorischen und persönlichen Schutzmaßnahmen.

Der Gesetzgeber hat mit der „Technischen Regel für Biologische Arbeitsstoffe“ (TRBA 250) Vorgaben zum Einsatz sicherer Instrumente festgelegt. Die Technischen Regeln für Biologische Arbeitsstoffe (TRBA) geben den Stand der sicherheitstechnischen, arbeitsmedizinischen, hygienischen sowie arbeitswissenschaftlichen Anforderungen bei Tätigkeiten mit Biologischen Arbeitsstoffen wieder. Sie werden vom Ausschuss für Biologische Arbeitsstoffe (ABAS) aufgestellt und von ihm der Entwicklung entsprechend angepasst. Die TRBA werden vom Bundesministerium für Wirtschaft und Arbeit im Bundesarbeitsblatt (seit 2007 im Gemeinsamen Ministerialblatt) bekannt gegeben, nach der sichere Arbeitsgeräte bei Tätigkeiten bzw. in Bereichen mit höherer Infektionsgefährdung oder Unfallgefahr einzusetzen sind [26].

Die Industrie bietet mittlerweile vielfältige Systeme für Injektionen, Infusionen und Blutentnahmen an, die bereits von der Konstruktion her das Risiko von Stichverletzungen erheblich verringern. $\mathrm{Zu}$ diesen sicheren Instrumenten zählen z.B. Injektionsnadeln, die sich nach Gebrauch in eine Schutzhülle zurückziehen, Injektionsnadeln mit leicht arretierbarem Stichschutz oder auch Injektionsnadeln, die beim Herausziehen stumpf werden [27]. Durch die Verwendung sicherer Instrumente konnte die Rate von NSV nachweislich gesenkt werden [10]. Des Weiteren kann speziell im OP das Verletzungsrisiko durch die Verwendung von Sicherheitsskalpellen gesenkt werden. Durch den Einzug neuer operativer, minimalinvasiver Techniken ist die Verletzungsgefahr ebenfalls deutlich gemindert worden.

Eine weitere, leicht umzusetzende Sicherheitsmaßnahme ist die Verwendung von stich- und bruchfesten Abwurfbehältern für das Sammeln von gebrauchten spitzen oder scharfen Gegenständen.

Neben Festlegung und Einhaltung von Hygienemaßnahmen entsprechend der gesetzlichen Vorschriften (TRBA 250, Biostoffverordnung) kann auch durch organisatorische Maßnahmen die Zahl der NSV verringert werden. Dazu zählen z. B. verbesserte Arbeitsanweisungen, regelmäßige Arbeitsschutzunterweisungen der Mitarbeiter, aber auch eine optimale Arbeitsplatzbeleuchtung und die ergonomisch günstige Aufstellung der Abwurfbehälter.

Von Fachgesellschaften werden sogenannte „neutrale Übergabezonen“ gefordert (z.B. Tücher oder Magnettafeln), damit es während der OP nicht zu Verletzungen bei der Instrumentenübergabe kommt [28].

Um Verletzungen infolge von Übermüdung zu vermeiden, ist eine gut durchdachte Schichtplangestaltung zu empfehlen, die nicht zuletzt durch angepasste Regelungen des Arbeitszeitgesetzes ermöglicht wird, da Übermüdung zu Unachtsamkeit und damit auch zur Häufung von Unfällen führt [29-31].

Das Tragen persönlicher Schutzausrüstungen (z.B. Handschuhe, Schutzmasken, Schutzkleidung) ist beim Umgang mit Patienten ein wesentlicher Sicherheitsstandard, der unbedingt befolgt werden sollte. Die Exposition gegenüber Körperflüssigkeiten während Operationen wird durch Tragen doppelter Handschuhe gemindert. Hier erlauben doppelte Handschuhe mit Indikatorfunktion die Entdeckung von Perforationen während operativer Eingriffe, sodass defekte Handschuhe sofort ersetzt werden können. Zudem reduzieren diese Handschuhsysteme das übertragene Blutvolumen deutlich, was ebenfalls zur Sicherheit des Operateurs beiträgt [32-34].

$\mathrm{Zu}$ den persönlichen Schutzmaßnahmen zählen des Weiteren Schutzimpfungen, die bei Beschäftigten im Gesundheitsdienst einen hohen Stellenwert einnehmen. Seit Anfang der 80er-Jahre ist die Schutzimpfung gegen Hepatitis B möglich. Alle Beschäftigten im Gesundheitswesen, bei denen Hepatitis B am Arbeitsplatz vorkommen kann, sollten einen aktuellen Impfschutz gegen Hepatitis B aufweisen. Dabei ist entsprechend der Impfempfehlungen der Ständigen Impfkommission am Robert Koch-Institut zu verfahren [35]. Bei korrekt durchgeführter Impfung sind bei mehr als 95\% der geimpften Personen Antikörper gegen HBs-Antigen nachweisbar.

Im Interesse sowohl der Mitarbeiter selbst als auch den Patienten gegenüber sollten invasive Tätigkeiten, bei denen eine erhöhte Verletzungsgefahr für das medizinische Personal besteht (z.B. bei Operationen in beengtem OP-Feld), nur von Personen durchgeführt werden, die nachweislich eine Immunität gegen Hepatitis B besitzen, entweder nach erfolgreicher Hepatitis-B-Schutzimpfung oder als Folge einer ausgeheilten Infektion [15]. Thomsen et al. verdeutlichten sehr nachdrücklich die Forderung an die Mitarbeiter im Gesundheitsdienst, sich impfen zu lassen: „Wer sich nicht rechtzeitig gegen Hepatitis B impfen lässt, handelt grob fahrlässig seinen Patienten, seinen Berufskollegen und sich selbst gegenüber." [36]

Für schwangere Mitarbeiterinnen gilt das Mutterschutzgesetz bzw. die Verordnung zum Schutze der Mütter am Arbeitsplatz. Dieses Gesetz sieht vor, dass werdende Mütter aufgrund des Infektionsrisikos und der Gefährdung des Ungeborenen nicht invasiv tätig sein dürfen. Aufgrund der heutzutage vorhandenen technischen Möglichkeiten des Arbeitsschutzes ist es durchaus zu verantworten, dass nach entsprechender individueller Gefährdungsbeurteilung durch den Arbeitsmediziner bzw. Betriebsarzt sowie unter Einbindung der schwangeren Mitarbeiterin in den Entscheidungsprozess die werdende Mutter ihre klinische Tätigkeit weiter fortführen kann und darf [25].

\section{Postexpositionsprophylaxe (PEP)}

Ist trotz Vorsichtsmaßnahmen eine Exposition erfolgt, sollten sowohl der Indexpatient als auch der Exponierte serologisch und gegebenenfalls molekularbiologisch nachuntersucht werden [23]. Hier steht die Frage, ob bei einem ernstzunehmenden Infektionsrisiko die Zustimmung des Indexpatienten erforderlich ist. Der klinische Alltag zeigt, dass hier über das Prozedere Unsicherheiten bestehen für den Fall, dass der Patient keine Zustimmung gibt [37]. Für die optimale Therapie des betroffenen Mitarbeiters ist die Testung des Indexpatienten bzw. die Kenntnis über den Infektionsstatus jedoch sinnvoll und notwendig. Vor einer Testung 
des Indexpatienten auf Infektionserkrankungen sollte dessen Zustimmung eingeholt werden. Für den Fall, dass der Indexpatient diese Zustimmung verweigert, hat der Arzt nach §34 StGB die Möglichkeit (und aus ethischer Sicht auch die Verpflichtung dem Mitarbeiter gegenüber), die Testung beim Patienten durchzuführen. Der Schutz des betroffenen Mitarbeiters ist als ein höheres Rechtsgut einzustufen als das Recht des Patienten auf „Nichtwissen“ seines Infektionsstatus [37].

\section{Hepatitis B}

Für das Vorgehen nach einer NSV mit Infektionsrisiko einer Hepatitis B wird ein differenziertes Vorgehen empfohlen. Falls nicht mit der Übertragung von HBV zu rechnen ist, sind keine speziellen Maßnahmen zur Verhütung einer Hepatitis-B-Infektion erforderlich. Allerdings sollte bei Personen ohne Immunität gegen Hepatitis B eine Immunisierung begonnen bzw. ggf. eine Auffrischimpfung - vom Betriebsarzt - durchgeführt werden.

Weitere Maßnahmen erübrigen sich ebenfalls, wenn der Beschäftigte immun oder durch Impfung ausreichend geschützt ist. Dies ist der Fall bei erfolgreicher Grundimmunisierung (anti-HBs M $100 \mathrm{IE} / \mathrm{l}$ innerhalb der letzten 12 Monate oder erfolgreiche Impfung innerhalb der vergangenen 5 Jahre). Liegt die erfolgreiche Grundimmunisierung mehr als 10 Jahre zurück, sind ebenfalls keine speziellen Maßnahmen bez. Hepatitis B erforderlich, wenn folgende Bedingungen erfüllt sind: Es liegt ein aktueller AntiHBs-Titer von $>100 \mathrm{IE} / \mathrm{l}$ vor bzw. bei der letzten betriebsärztlichen Untersuchung (die nicht länger als 3 Jahre zurückliegt) wurde ein ausreichender Schutz gegen Hepatitis B festgestellt (es erfolgte keine Empfehlung zur Booster-Impfung). Die sofortige Testung des Verletzten ist bei unvollständiger Immunisierung, bei „Nonrespondern“ oder bei fehlender Impfkontrolle zu veranlassen: Bei Anti-HBs-Titer > $100 \mathrm{IE} / \mathrm{l}$ sind keine speziellen Maßnahmen bez. Hepatitis B erforderlich, bei Anti-HBs-Titern $<100 \mathrm{IE} / \mathrm{l}$ muss eine Booster-Impfung mit Aktivimpfstoff erfolgen [35]. Für bisher nicht geimpfte Personen steht eine aktive und passive Immunprophylaxe zur Verfügung. Die Empfehlungen der Ständigen Impfkommission [13] zur Postexpositionsprophylaxe nach NSV sind in 0 Tab. 1 zusammengefasst.

Liegt das Ereignis (z. B. Nadelstichverletzung) länger als 48 h zurück, so empfiehlt das Kompetenznetz Hepatitis vor der Gabe von Immunglobulin die Rücksprache mit einem hepatologischen Zentrum oder mit dem Kompetenznetz Hepatitis selbst. Unabhängig davon ist aber unbedingt eine Aktivimpfung durchzuführen [38]. Des Weiteren sollten jeweils nach 12 und 26 Wochen Kontrolluntersuchungen erfolgen. Hier wird die Untersuchung von GOT und GPT sowie Anti-HBc empfohlen. Darüber hinaus ist nach einer NSV die Anzeige auf Verdacht einer Berufskrankheit beim zuständigen Gewerbearzt oder der zuständigen Berufsgenossenschaft erforderlich.

\section{Hepatitis C}

Im Falle einer beruflichen HCV-Exposition (z. B. nach Nadelstichverletzung) steht derzeit keine Postexpositionsprophylaxe zur Verfügung. Unmittelbar nach der Verletzung/Kontamination sollten beim Exponierten Anti-HCV und ALT bestimmt werden (Ausgangswert). Im Verlauf sollte nach 2-4 Wochen eine Bestimmung der HCV-RNA erfolgen. Falls negativ, kann diese Untersuchung 68 Wochen nach Exposition wiederholt werden. Bei Nachweis einer akuten Infektion sollte eine Therapie mit Interferon alpha oder pegyliertem Interferon alpha zur Verhinderung einer Chro-
Tab. 1 Empfehlungen der STIKO für die Postexpositionsprophylaxe nach Kontakt mit HBV.

\begin{tabular}{|lll|}
$\begin{array}{ll}\text { aktueller Anti-HBs-Wert } \\
\text { des Exponierten }\end{array}$ & HB-Impfstoff & HB-Immunglobulin \\
$>100 \mathrm{IE} / \mathrm{I}$ & nein & nein \\
\hline $10-100 \mathrm{IE} / \mathrm{l}$ & ja & nein \\
\hline$<10 \mathrm{IE} / \mathrm{j}$ & ja & ja \\
\hline nicht zu bestimmen & ja & ja \\
\hline
\end{tabular}

nifizierung eingeleitet werden. Die antivirale Therapie einer akuten HCV-Infektion sollte über 24 Wochen durchgeführt werden $[38,39]$.

\section{HIV}

Nach Verletzungen mit HIV-kontaminierten Instrumenten steht eine medikamentöse Therapie zur Verfügung. Eine Empfehlung zur Prophylaxe sollte der Patient bei tiefen Stich- oder Schnittverletzungen, sichtbaren Blutspuren auf dem verletzenden Instrument, perkutaner Verletzung mit Injektionsnadel oder anderer Hohlraumnadel sowie bei hoher Viruslast im Blut des Indexpatienten erhalten. Die Therapie sollte so früh wie möglich beginnen (möglichst innerhalb von $24 \mathrm{~h}$, besser noch innerhalb von $2 \mathrm{~h}$ ) und in der Regel über 28 Tage erfolgen. Die Standardprophylaxe wird entweder mit der Kombination aus 2 Inhibitoren der reversen Transkriptase (RTI) und einem Proteaseinhibitor (PI) durchgeführt oder aber mit der Kombination aus 2 RTI und einem nicht nukleosidalen Reverse-Transkriptase-Inhibitor (NNRTI) [22].

Unabhängig von den medikamentösen Maßnahmen sind folgende Schritte unverzüglich einzuleiten:

- bei Stich- und Schnittverletzungen sollte der Blutfluss durch Druck auf das umliegende Gewebe gefördert werden,

- antiseptische Spülung und Anlegen eines antiseptischen Wirkstoffdepots auf der Basis von PVP-Jod/Alkohol,

- Untersuchung des Indexpatienten,

- Einleitung eines D-Arzt-Verfahrens,

- Vorstellung beim Betriebsarzt.

Wie das Regeluntersuchungsprogramm nach Stich- und Schnittverletzungen konkret durchzuführen ist, hat die BGW in einer Broschüre zusammengefasst [40].

\section{Fazit}

$\nabla$

Die vordringlichen Aufgaben des Arbeitsmediziners bzw. Betriebsmediziners liegen in der Prävention. Als Fazit ist hier festzuhalten, dass es zahlreiche präventive Maßnahmen gibt, die Verletzungen an spitzen und scharfen Gegenständen zu mindern bzw. zu verhindern helfen. Die Verwendung von sicheren Instrumenten bei invasiven Eingriffen, verbesserte organisatorische Maßnahmen sowie das Verwenden persönlicher Schutzausrüstungen (z. B. das Tragen doppelter Handschuhe) für eine verstärkte Sicherheit sollten zur Selbstverständlichkeit beim medizinischen Personal nicht nur im operativen Bereich werden.

Interessenkonflikt: Nein 


\section{Literatur}

1 Wittmann A, Zeljka V, Neukirch B et al. Kosten durch Nadelstichverletzungen. ErgoMed/Prakt Arbmed 2011; 35: 8-12

2 Zschernack S, Göbel M, Friesdorf W. Arbeits- und Gesundheitsschutz im Operationssaal. In: Badura B, Schellschmidt H, Vetter C, Hrsg. Fehlzeitenreport 2004. Berlin, Heidelberg, New York: Springer; 2004

3 Wicker S, Rabenau HF. Nadelstichverletzungen im klinischen Alltag. Ergebnisse der Frankfurter Nadelstichstudie. Trauma Berufskrankh 2008; 10 (Suppl 1): 64-68

4 Wicker S, Rabenau HF. Nadelstichverletzungen bei Mitarbeitern im Gesundheitswesen: Berufsrisiko oder vermeidbare Infektionsgefährdung? Ergebnisse der Frankfurter Nadelstichstudie. Krh.-Hyg. + Inf. Verh. 2007; 29: 86-90

5 Thalhammer $F$. Nadelstichverletzungen - wie häufig sind sie wirklich? Wien Klin Wochenschr 2008; 120: 458-460

6 http://www.dguv.de/inhalt/zahlen/documents/bk-dok-2008.pdf; Stand: 12.03 .2012

7 http://www.dguv.de/inhalt/zahlen/documents/dguvstatistiken2010d.pdf; Stand: 12.03 .2012

8 Rabenau HF, Wicker S. Gefährdungspotential durch Nadelstichverletzungen: Eine internationale Herausforderung. Krh.-Hyg. + Inf.verh. 2007; 29: 82-85

9 Wicker S, Gottschalk R, Rabenau HF. Gefährdungen durch Nadelstichverletzungen. Betrachtung aus arbeitsmedizinischer und virologischer Sicht. Dtsch Arztebl 2007; 104: A3102-A3107

10 Hofmann F, Kralj N, Beie M. Kanülenstichverletzungen im Gesundheitsdienst - Häufigkeit, Ursachen und Präventionsstrategien. Gesundheitswesen 2002; 64: 259-266

11 Wicker S, Rabenau HF, Groneberg DA et al. Arbeitsbedingte Infektionen bei Mitarbeitern des Gesundheitswesens: Blutübertragbare Erkrankungen. Zbl Arbeitsmed 2009; 59: 138-150

12 http://www.so.ch/fileadmin/internet/ddi/ighaa/pdf/kaed/ Infektionskrankheiten/Verhuetung_blutuebertragbarer_ Krankheiten_SUVA.pdf; Stand: 22.03.2012

13 http://www.rki.de/cln_153/nn_468102/DE/Content/Infekt/EpidBull/ Merkblaetter/Ratgeber_HepatitisB.html; Stand: 13.03.2012

14 Hofmann F, Wittmann A, Kralj N. Risikoabschätzung nach arbeitsmedizinisch relevanten Nadelstichverletzungen durch Messen des übertragenen Blutvolumens. Arbeitsmed Sozialmed Umweltmed 2004, 39: 206

15 RKI. Zur Situation bei wichtigen Infektionskrankheiten in Deutschland. Epidemiologisches Bulletin 2011; 29: 261-274

16 Laufs $R$, Polywka S, Feucht HH et al. Hepatitis C. Virologie, Übertragungswege, Klinik, Prävention und Therapie. Anaesthesist 2002; 51: 884-889

17 Müller R. Hepatitis C. Die Erkrankung und ihre Konsequenzen. Trauma Berufskrankh 2008; 10: 61-63

18 Kubitschke A, Bader C, Tillmann HL et al. Verletzungen mit Hepatitis-CVirus-kontaminierten Nadeln. Internist 2007; 48: 1165-1172

19 Bäuerle V, Nowak D. Hepatitis C aus arbeitsmedizinischer Sicht. Anaesthesist 2003; 52: 459-464

20 Sarazzin C, Berg T, Ross RS et al. Update der S3-Leitlinie Prophylaxe, Diagnostik und Therapie der Hepatitis-C-Virus (HCV)-Infektion, AWMF-Register-Nr.: 021/012. Z Gastroenterol 2010; 48: 289-351

21 Yazdanpanah Y, De Carli G, Migueres B et al. Risk factors for hepatitis C virus transmission to health care workers after occupational exposure: A European case-control-study. Clin Infect Dis 2005; 41: 1423-1430

22 RKI. Zum Welt-AIDS-Tag. Epidemiologisches Bulletin 2011; 46: 416428
23 Sarrazin $U$, Brodt, $R$, Sarrazin $C$ et al. Postexpositionsprophylaxe nach beruflicher Exposition mit HBV, HCV und HIV. Unfallchirurg 2004; 107: $129-142$

24 Gerberding JL. Incidence and prevalence of human immunodeficiency virus, hepatitis B virus, hepatitis C virus, and cytomegalovirus among health care personal at risk for blood exposure: Final report from a longitudinal study. J Infect Dis 1994; 170: 1410-1417

25 Wicker S, Rabenau HF, Haberl AE et al. Blutübertragbare Infektionen und die schwangere Mitarbeiterin im Gesundheitswesen. Risiko und Präventionsmaßnahmen. Chirurg 2012; 83: 136-142

26 Technische Regel für Biologische Arbeitsstoffe 250; Biologische Arbeitsstoffe im Gesundheitswesen und in der Wohlfahrtspflege (TRBA 250) Ausgabe: November 2003; zuletzt geändert und ergänzt: GMBI. Nr. 4 vom 14. Februar 2008, S. 83

27 Haamann F. Liste sicherer Produkte - Schutz vor Schnitt- und Stichverletzungen 2008. Erstveröffentlichung 11/2008, Stand 02/2012. Berufsgenossenschaft für Gesundheitsdienst und Wohlfahrtspflege - BGW: 2012

28 http://www.facs.org/fellows_info/statements/st-58.html; Stand: 14.05 .2012

29 Fishman DN, Harris AD, Rubin $M$ et al. Fatigue increases the risk of injury from sharp devices in medical trainees: results from a case-control study. Infect Control Hosp Epidemiol 2007; 28: 10-17

30 Ilhan MN, Durukan E, Aras E et al. Long working hours increase the risk of sharp and needlestick injury in nurses: the need for new policy implication. J Adv Nurs 2006; 56: 563-568

31 Arbeitsmedizinische Leitlinie der Deutschen Gesellschaft für Arbeitsmedizin und Umweltmedizin e.V. Nacht- und Schichtarbeit. Arbeitsmed. Sozialmed. Umweltmed. 2006; 41: 390-397

32 FitzSimons D, François G, De Carli G et al. Hepatitis B virus, hepatitis C virus and other bloodborne infections in healthcare workers: guidelines for prevention and management in industrialised countries. Occup Environ Med 2008; 65: 446-451

33 Wittmann A, Kralj N, Köver J et al. Study of blood contact in simulated surgical needlestick injuries with single or double latex gloving. Infect Control Hosp Epidemiol 2009; 30: 53-56

34 Kinlin LM, Mittleman MA, Harris AD et al. Use of gloves and reduction of risk of injury caused by needles or sharp medical devices in healthcare workers: results from a case-crossover study. Infect Control Hosp Epidemiol 2010; 31: 908-917

35 RKI. Mitteilung der Ständigen Impfkommission am Robert Koch-Institut. Epidemiologisches Bulletin 2011; 30: 275-294

36 Thomsen R. Hepatitis B. Der HBV-infizierte Chirurg - ein Infektionsrisiko für den Patienten. Chirurg 2002; 73: 435-438

37 Wicker S, Rabenau HF, Marckmann G et al. Blutuntersuchung nach Nadelstichverletzung: Ist die Zustimmung des Indexpatienten erforderlich? Arbeitsmed Sozialmed Umweltmed 2009; 44: 301-303

38 http://www.kompetenznetz-hepatitis.de/; Stand: 13.03 .2012

39 http://www.rki.de/cln_234/nn_468106/DE/Content/Infekt/EpidBull/ Merkblaetter/Ratgeber_HepatitisC.html; Stand: 12.03.02012

40 Hamann F. Vorgehen nach Stich- und Schnittverletzungen - Begründung für das Regeluntersuchungsprogramm der BGW. Stand 2/2008. Berufsgenossenschaft für Gesundheitsdienst und Wohlfahrtspflege - BGW. www.bgw.online.de \& http://www.bgw-online.de/internet/ generator/Inhalt/Onlinelnhalt/Medientypen/Fachartikel/ Regeluntersuchungsprogramm__Nadelstichverletzungen,property= pdfDownload.pdf; Stand: jeweils 22.03.2012 